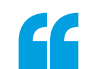

Interestingly, this mechanism could theoretically work in bulk glass also

$\Rightarrow$ GLASSES

\title{
A flawless flow
}

Inorganic oxide glasses are typically brittle at room temperature, which limits their use in real-life settings. However, their properties are wide-ranging - from semiconducting to insulating making them attractive components for electronic devices and batteries. Thus, researchers are keen to make oxide glasses that can deform in a plastic manner and to realize damage-tolerant glasses.

Now, writing in Science, Erkka Frankberg, Lucile Joly-Pottuz and colleagues report thin films of an amorphous aluminium oxide that are highly ductile at room temperature under shear and tensile loading. This ductility allows the flexible oxide glass to be bent and pulled,

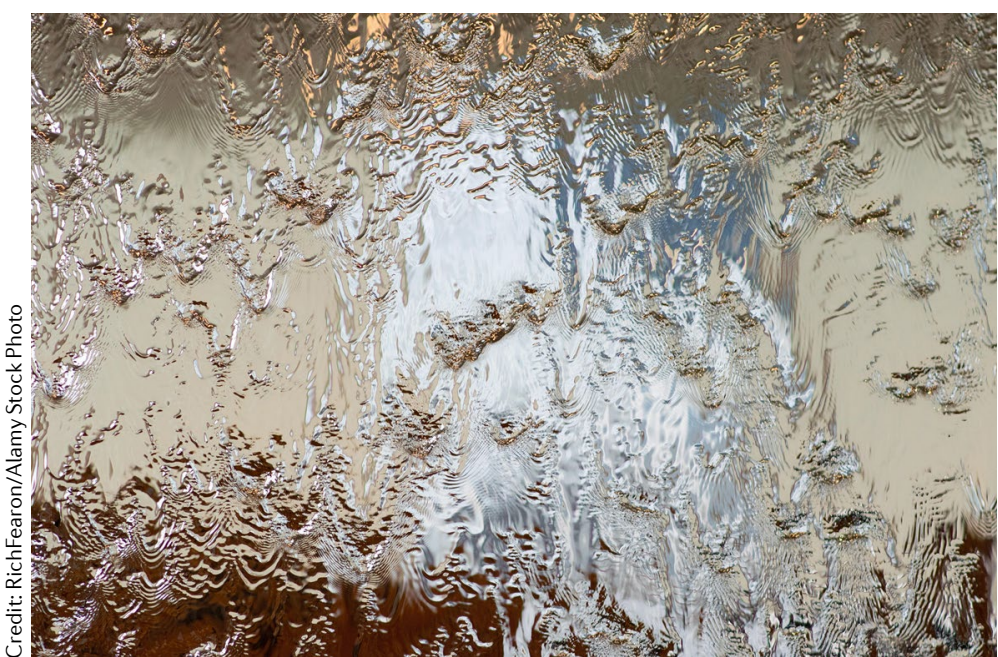

and hence permanently deformed without breaking. "Moreover, as a result of the plasticity, the glass exhibits a very high strength to weight ratio," says Frankberg.

The plastic behaviour arises owing to a viscous creep mechanism which usually requires high temperatures to occur in other oxide glasses. "Although this plasticity mechanism is different to metals, we found that a similar magnitude of plastic strains can be accommodated by the aluminium oxide glass," says Frankberg. A total elongation of $100 \%$ is possible for the glass.

The viscous flow relies on the fabrication of thin films of aluminium oxide that are dense and flaw-free; the absence of defects allows sufficient bond-switching to enable the rearrangement of atoms such that the material can flow. In addition, the activation energy of the bond-switching process has to be sufficiently low for flow to occur. "Interestingly, this mechanism could theoretically work in bulk glass also," says Frankberg. "Based on our results, the main obstacle seems to be the manufacturing of flaw-free bulk glass."

Making thin films without flaws is not straightforward, and for applications it is likely that large-area films, or thicker films, will be a necessity. The challenge now is to make such films and, in part, this preparation will also require the use of characterization techniques that can detect nanoscale defects in the material.

In the future, the plastic-like amorphous glass may be incorporated in device settings where damage-tolerant glass is needed. "Based on these results, it could be possible to substantially increase the mechanical resilience of everyday electronic appliances," says Frankberg. "For example, glass covers of smart phones could be made to withstand severe impacts or car batteries could be made to withstand mechanical damage that otherwise would lead to catastrophic short circuit. In more advanced applications, space solar cells could be protected from meteoroids and space debris."

\section{Alison Stoddart}

ORIGINAL ARTICLE Frankberg, E.J. et al. Highly ductile amorphous oxide at room temperature and high strain rate. Science 366, 864-869 (2019) 